\title{
3D Relativistic MHD Simulations of Magnetized Spine-Sheath Relativistic Jets
}

\author{
Y. Mizuno $^{* a}$, P. Hardee ${ }^{b}$, and K.-I. Nishikawa ${ }^{a}$ \\ ${ }^{a}$ National Space Science and Technology Center \\ 320 Sparkman Drive, VP 62, Huntsville, AL 35805, USA \\ E-mail: Yosuke.Mizuno@msfc.nasa.gov \\ ${ }^{b}$ Department of Physics and Astronomy, The University of Alabama \\ Tuscaloosa, AL 35487, USA
}

\begin{abstract}
We have performed numerical simulations of weakly and strongly magnetized relativistic jets embedded in a weakly and strongly magnetized stationary or mildly relativistic $(0.5 c)$ sheath using the RAISHIN code. In the numerical simulations a jet with Lorentz factor $\gamma=2.5$ is precessed to break the initial equilibrium configuration. Results of the numerical simulations are compared to theoretical predictions from a normal mode analysis of the linearized RMHD equations describing a uniform axially magnetized cylindrical relativistic jet embedded in a uniform axially magnetized moving sheath. The prediction of increased stability of a weakly-magnetized system with mildly relativistic sheath flow to Kelvin-Helmholtz instabilities and the stabilization of a strongly-magnetized system with mildly relativistic sheath flow is confirmed by the numerical simulations.
\end{abstract}

VI Microquasar Workshop: Microquasars and Beyond

September 18-22 2006

Società del Casino, Como, Italy

\footnotetext{
*Speaker.
} 


\section{Introduction}

Relativistic jets have been observed in galaxies and quasars (AGNs)[1, 2], in black hole binary star systems (microquasars)[3], and are thought responsible for the gamma-ray bursts (GRBs)[5]. Proper motions observed in microquasar and AGN jets imply the jet speeds from $\sim 0.9 c$ up to $\sim 0.999 c$.

While jets at the larger scales may be kinetically dominated and contain relatively weak magnetic fields, the possibility of much stronger magnetic fields certainly exists closer to the acceleration and collimation region. Recent GRMHD simulations of jet formation performed by Mizuno et al. (2006a) [6] indicate that highly collimated high speed jets driven by the magnetic fields threading the ergosphere may themselves reside with a broader wind or sheath outflow driven by the magnetic fields anchored in the accretion disk. This configuration might additionally be surrounded by a less collimated accretion disk wind from the hot corona [7].

Recent observations of high speed winds in several QSO's (0.1 - $0.4 \mathrm{c})$ [8] also indicate that the highly relativistic jet could reside in a high speed wind or sheath, at least close to the central engine. Other observational evidence such as limb brightening has been interpreted as evidence for a slower external flow surrounding a faster jet spine [9]. A spine-sheath jet structure has also been proposed based on theoretical arguments [10].

In this paper we investigate the stability properties of highly relativistic jet flows allowing for the effects of strong magnetic fields and relativistic flow in a sheath around the highly relativistic jet by 3D RMHD numerical simulations. Such 3D numerical simulations of fluid relativistic jets and accompanying theoretical work has allowed relatively unambiguous interpretation of the structures observed in the numerical simulations[11, 12, 13].

\section{Numerical Setup}

In order to study the long-term stability of magnetized sheath-spine relativistic jets, we use the 3-dimensional GRMHD code "RAISHIN" with Cartesian coordinates in special relativity. The method is based on a $3+1$ formalism of the general relativistic conservation laws of particle number and energy momentum, Maxwell equations, and Ohm's law with no electrical resistance (ideal MHD condition) on a curved spacetime [14]. The RAISHIN code can perform special relativistic computations in Minkowski spacetime by changing the metric.

We consider the following initial conditions for the simulations: "preexisting" jet flow is established across the computational domain. This represents the case in which a leading Mach disk and bow shock has passed. The jet flow is surrounded by a low-density external wind (medium). For all simulations, the ratio of densities is $\rho_{j} / \rho_{e}=2.0$, where $\rho$ is the mass density in the proper frame. The jet flow has $v_{j}=0.9165 c$ and $\gamma \equiv\left(1-v^{2}\right)^{-1 / 2}=2.5$. The initial magnetic field is assumed to be uniform and parallel to the jet flow. We have performed two sets of simulations. In the weakly magnetized (RHD) simulations, the relevant sound speeds are $a_{e}=0.574 c$ and $a_{j}=0.511 c$, the relevant Alfvén speeds are $v_{A e}=0.0682 c$ and $v_{A j}=0.064 c$, and the Alfvén speed is much smaller than sound speed. In the strongly magnetized (RMHD) simulations. The relevant sound speeds are $a_{e}=0.30 c$ and $a_{j}=0.226 c$, the relevant Alfvén speeds are $v_{A e}=0.56 c$ and $v_{A j}=0.45 c$, and the Alfvén speed is comparable to the sound speed. In order to investigate the effect of an external 


\section{RHD, no wind, $\omega=0.93$, time $=60.0$}
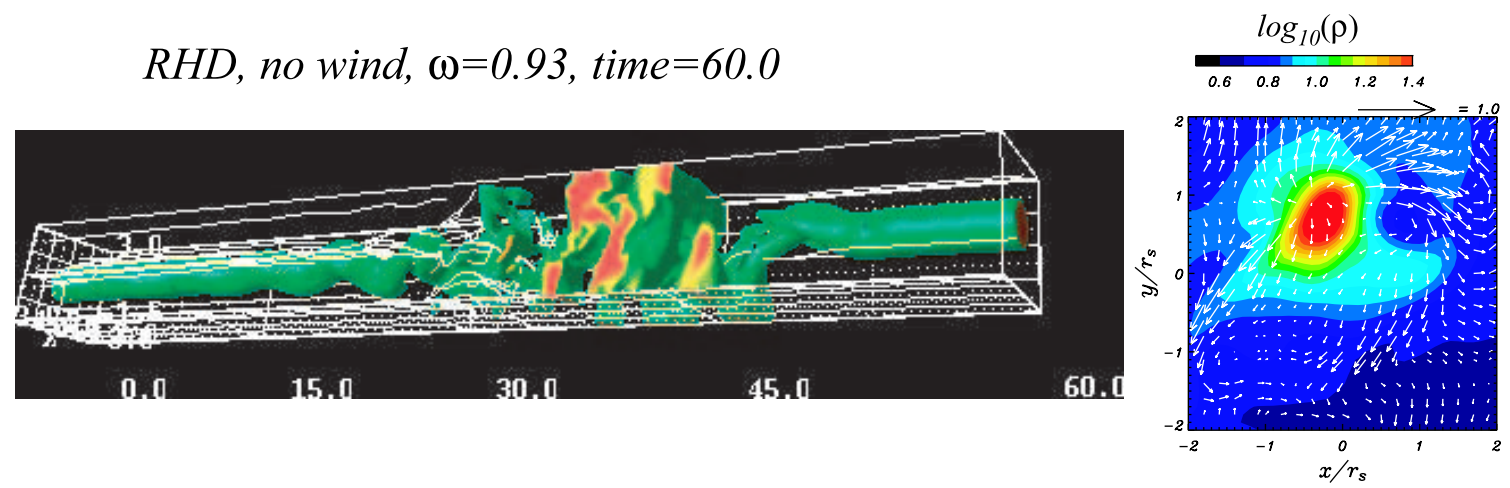

Figure 1: Three-dimensional isovolume image $(a)$ and transverse $(b)$ cut $\left(z=30 R_{j}\right.$ ) of the weakly magnetized case without an external wind. The color scales show the logarithm of density. The white lines indicate magnetic field lines. The arrows depict transverse velocities.

wind, we have performed simulations with no external wind $\left(v_{e}=0.0 c\right)$ and a mildly relativistic external wind $\left(v_{e}=0.5 c\right)$ for both weakly- and strongly-magnetized cases. The computational domain is $3 R_{j} \times 3 R_{j} \times 60 R_{j}$ with $60 \times 60 \times 600$ computational zones ( 10 computational zones span $R_{j}$ ). We impose outflow boundary conditions on all surfaces except the inflow plane, $z=0$. A precessional perturbation of angular frequency $\omega R_{j} / v_{j}=0.93$ is applied at the inflow by imposing a transverse component of velocity with $v_{\perp}=0.01 v_{j}$. The simulations are halted after $\sim 60$ light crossing times of the jet radius, and before the perturbation has crossed the entire computational region.

\section{Results}

Figure 1 shows the structure of a weakly magnetized jet (three-dimensional isovolume image and transverse cuts) without an external wind at $t=60$. The precession at the jet inflow plane excites the Kelvin-Helmholtz (KH) instability. The initial perturbation propagate down the jet and grows as a helical structure. Beyond $R_{j}=40$ the $\mathrm{KH}$ instability disrupst the jet structure strongly. The magnetic field is strongly distorted and becomes complicated. The transverse cut at $z=30 R_{j}$ seen Fig $1 \mathrm{~b}$, shows strong interaction with the external medium at this distance. Transverse velocities show circular motions near the jet surface caused by the helically twisted jet.

To investigate simulation results quantitatively, we take one-dimensional cuts through the computational box parallel to the $\mathrm{z}$-axis at different radial distances along the transverse $\mathrm{x}$-axis at $x / R_{j}=0.2,0.5$, and 0.8, as shown in Figure 2 .

The weakly magnetized simulation results show velocity oscillation from the growing helical $\mathrm{KH}$ instability. The simulations are fully evolved to a non-linear phase by about $32 R_{j}$ for no external wind and beyond about $38 R_{j}$ for an external wind. The external wind has reduced the growth of $\mathrm{KH}$ instability and has delayed the onset of the non-linear phase. The dominant wavelength of oscillation is $\lambda / R_{j} \sim 6$ in both weakly magnetized cases. The strongly magnetized simulation results without an external wind also show growing velocity oscillation (Figure 2c), albeit more slowly. The simulation is still evolving linearly and has not reached the non-linear phase. From the comparison with the weakly-magnetized cases, the stronger magnetic field reduces the growth 

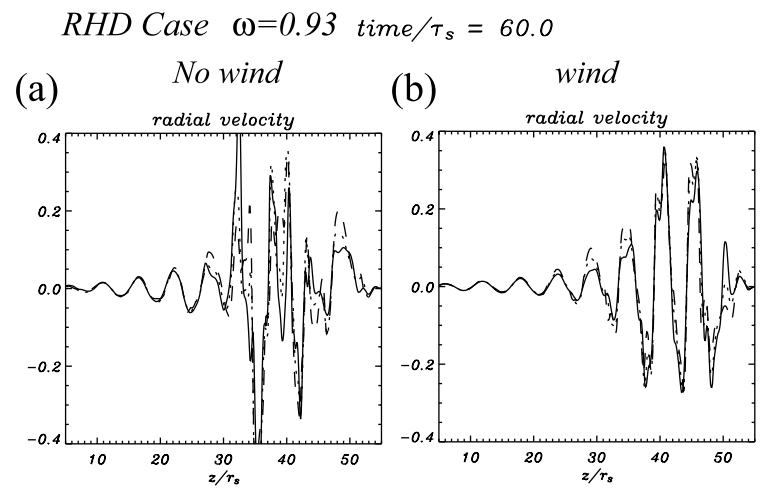

RMHD Case $\omega=0.93$ time $/ \tau_{s}=60.0$

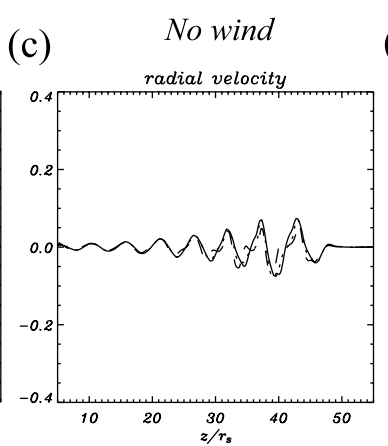

(d) wind

Figure 2: Radial velocity $\left(v_{x}\right)$ along the one-dimensional cuts parallel to the jet axis located at $x / R_{j}=0.2$ (solid line), 0.5 (dotted line) and 0.8 (dashed line) for the weakly $(a, b)$ and strongly $(c, d)$ magnetized cases without an external wind $(a, c)$ and with an external wind $(b, d)$.

rate of the helical KH instability. The strongly magnetized simulation result with an external wind reveals a damped oscillation. Therefore the external wind in the strongly magnetized case leads to damping of KH instability and stabilizes the jet. The dominant oscillation wavelength is $\lambda / R_{j} \sim 5$ in both of the strongly magnetized cases.

The simulations confirm theoretical predictions for the stability of a jet spine-sheath or jetwind configuration Hardee (2006) [15]. Theoretical predictions arise from a normal mode analysis of the linearized ideal RMHD equations describing a uniform axially magnetized cylindrical relativistic jet embedded in a uniform axially magnetized moving sheath. The most important theoretical prediction obtained from the normal mode analysis is that the presence of an external wind and strong magnetic field can reduce the growth rate or lead to damping of the $\mathrm{KH}$ instability. This prediction is confirmed by the present simulation results.

\section{Conclusion}

We have performed numerical simulations of weakly and strongly magnetized relativistic jets embedded in a weakly and strongly magnetized stationary or mildly relativistic $(0.5 c)$ sheath using the RAISHIN code [14]. In the numerical simulations a jet with Lorentz factor $\gamma=2.5$ is precessed to break the initial equilibrium configuration. Results of the numerical simulations have been compared to theoretical predictions from a normal mode analysis of the linearized RMHD equations describing a uniform axially magnetized cylindrical relativistic jet embedded in a uniform axially magnetized moving sheath. The prediction of increased stability of weakly-magnetized systems with mildly relativistic sheath flow to Kelvin-Helmholtz instability and the stabilization of a strongly-magnetized system with mildly relativistic sheath flow is verified by the numerical simulation results.

\section{Acknowledgments}

Y. M. is a NASA Postdoctoral Program fellow at NASA Marshall Space Flight Center. K. N. is partially supported by the National Science Foundation awards ATM-0100997, INT-9981508, and 
AST-0506719, and the National Aeronautic and Space Administration award NASA-INTEG040000-0046 to the Univ of Alabama in Huntsville. P.H. acknowledges partial support by National Space Science and Technology (NSSTC/NASA) cooperative agreement NCC8-256 and NSF award AST-0506666. The simulations have been performed on the IBM p690 at the National Center for Supercomputing Applications (NCSA) which is supported by the NSF and Altix3700 BX2 at YITP in Kyoto University.

\section{References}

[1] C.M. Urry, \& P. Padovani, Unified Schemes for Radio-Loud Active Galactic Nuclei, PASP 107 (803) 1995.

[2] A. Ferrari, Modeling Extragalactic Jets, ARAA 36 (539) 1998.

[3] I.F. Mirabel, \& L.F. Rodríguez, Sources of Relativistic Jets in the Galaxy, ARAA 37 (409) 1999.

[4] T. Piran, The physics of gamma-ray bursts, Reviews of Modern Physics, 76 (1143) 2005.

[5] P. Mészáros, Gamma-ray bursts, Rep. Prog. Phys. 69 (2259) 2006.

[6] Y. Mizuno, K.-I. Nishikawa, S. Koide, P. Hardee, \& G.J. Fishman, General Relativistic Magnetohydrodynamic Simulations of Jet Formation with a Thin Keplerian Disk, ApJL 2006a submitted.

[7] K.-I. Nishikawa, G. Richardson, S. Koide, K. Shibata, T. Kudoh, P. Hardee, \& G.J. Fishman, A General Relativistic Magnetohydrodynamic Simulation of Jet Formation, ApJ 625 (60) 2005.

[8] K. Pounds, \& K. Page, Evidence for massive ionised outflows in (super-Eddington?) AGN, Nuc. Phys. B Proc. Sup. 132 (107) 2004.

[9] G. Giovannini, Observational Properties of Jets in Active Galactic Nuclei, Ap\&SS 293 (1) 2004.

[10] G. Henri, \& G. Pelletier, Relativistic electron-positron beam formation in the framework of the two-flow model for active galactic nuclei, ApJL 383 (L7) 1991.

[11] P.E. Hardee, P.A. Hughes, A. Rosen, \& E. Gomez, Relativistic Jet Response to Precession and Wave-Wave Interactions, ApJ 555 (744) 2001.

[12] I. Agudo, et al. Jet Stability and the Generation of Superluminal and Stationary Components, ApJL 549 (L183) 2001.

[13] P.E. Hardee, \& P.A. Hughes, The Effect of External Winds on Relativistic Jets, ApJ 583 (116) 2003.

[14] Y. Mizuno, K.-I. Nishikawa, S. Koide, P. Hardee, \& G.J. Fishman, RAISHIN: A High-Resolution Three-Dimensional General Relativistic Magnetohydrodynamics Code, ApJS 2006b submitted.

[15] P.E. Hardee, The Stability Properties of Strongly Magnetized Spine-Sheath Relativistic Jets, 2006 in preparation 